\title{
Comparative Study of Effect of Continuous versus Intermittent Subglottic Suctioning by the Suction Above the Cuff Endotracheal Tube (SACETT) on Tracheal Mucosa and Incidence of VAP in Intensive Care Unit
}

\author{
Ravi Anand ${ }^{1}$ Siddhart Singh ${ }^{2} \quad$ Anand Prasoon ${ }^{1}$ \\ ${ }^{1}$ Department of Trauma and Emergency, Indira Gandhi Institute of \\ Medical Sciences, Patna, Bihar, India \\ ${ }^{2}$ Department of Critical Care Medicine, Indira Gandhi Institute of \\ Medical Sciences, Patna, Bihar, India
}

J Card Crit Care 2021;5:196-200.

\author{
Sanjeev Kumar ${ }^{2}$ Ritu Singh ${ }^{2}$ Santosh Kumar Nayan ${ }^{1}$
}

Address for correspondence Ravi Anand, DNB, Department of Trauma and Emergency, Indira Gandhi Institute of Medical Sciences, Patna 800014, Bihar, India (e-mail: drravianand75@yahoo.com).

\begin{abstract}
Keywords

- intensive care

- intubation

- mechanical ventilation

- subglottic secretion drainage

- tracheal injury

- ventilator-associated pneumonia
\end{abstract}

Context Ventilator-associated pneumonia (VAP) is one of the important causes of morbidity and mortality in patients admitted to the intensive care unit (ICU) with ventilatory support. Subglottic secretion and their aspirations are primarily responsible for the occurrence of VAP and the risk of tracheal mucosa injury induced by subglottic suctioning and the risk of VAP inpatient on a ventilator has been raised.

Aims Aim of this study is to compare the effect of continuous suctioning of subglottic secretions (CSSSs) versus intermittent suctioning of subglottic secretions (ISSS) on tracheal mucosa damage and incidence of VAP inpatient on a ventilator in both study groups.

Settings and Design A prospective randomized study.

Methods and Material A total of 140 patients requiring intubation or reintubation in the ICU with an expected ventilation duration $>24$ hours were included in two groups: CSSS and ISSS. The effect on tracheal mucosa in front of the suction port was assessed after intubation (T0) and before extubation ( $\mathrm{T} 1$ ) by bronchoscopy. VAP was compared in both groups on the basis of clinical suspicion and positive culture of bronchoalveolar lavage.

Statistical Analysis Used The SAS statistical software version 9.4 (SAS Institute, Cary, North Carolina, United States) has been used for analysis. Chi-square of the Fisher's exact test and Student's $t$-test or the Wilcoxon rank-sum test were used for analysis. Results A total of 127 patients were included and 89 patients (CSSS, $n=44$ and ISSS, $n=45$ ) were evaluable on the primary endpoint. The occurrence or worsening of tracheal mucosal damages did not differ between the two groups (CSSS, $n=12$ [27.3\%] vs. ISSS, $n=8[17.7 \%], p=0.365)$. Daily average volume of suctioned secretion was
DOI https://doi.org/ $10.1055 / \mathrm{s}-0041-1741524$. ISSN 2457-0206.
(C) 2022. Official Publication of The Simulation Society (TSS), accredited by International Society of Cardiovascular Ultrasound (ISCU). All rights reserved.

This is an open access article published by Thieme under the terms of the Creative Commons Attribution-NonDerivative-NonCommercial-License, permitting copying and reproduction so long as the original work is given appropriate credit. Contents may not be used for commercial purposes, or adapted, remixed, transformed or built upon. (https://creativecommons.org/ licenses/by-nc-nd/4.0/)

Thieme Medical and Scientific Publishers Pvt. Ltd., A-12, 2nd Floor, Sector 2, Noida-201301 UP, India 
higher with ISSS ( $72 \pm 100$ vs. $18 \pm 25 \mathrm{~mL}, p<0.001$ ). Negligible chance to aspirate was higher with CSSS $(0.13 \pm 0.15$ vs. $0.03 \pm 0.06$ per day, $p<0.001)$.

Conclusion ISSS did not reduce mucosal damage as compared with CSSS. The aspirated volume was higher and the negligible chance to aspirate was lower with ISSS. The incidence of VAP was lower with CSSS.

\section{Introduction}

Ventilator-associated pneumonia (VAP) is one of the important causes for morbidity and mortality in patients admitted to the intensive care unit (ICU) with ventilatory support. Subglottic secretion and their aspiration is primarily responsible for the occurrence of VAP. ${ }^{1}$

The subglottic secretion drainage above the cuff of an endotracheal (ET) tube is identified as an effective method to prevent VAP in critically ill patients, ${ }^{2-4}$ But risk of tracheal mucosa damage induced by suctioning in critically ill patients is a matter of concern. ${ }^{5,6}$ The aim of this study was to compare the incidence of ET damage induced by suctioning with the latest generation of ET tube (Suction Above the Cuff Endotracheal Tube [SACETT]) and to compare the effect of continuous suctioning of subglottic secretions (CSSS) or intermittent suctioning of subglottic secretions (ISSS) on tracheal mucosa damage in intensive care-ventilated patients and to compare the incidence of VAP in both groups.

\section{Materials and Methods}

After approval from institutional ethics committee of Indira Gandhi Institute of Medical Sciences, Patna, Bihar, India (date of acceptance 23/03/2021 letter no. 83/IEC/IGIMS/2021), patients were selected for the single-blinded, randomized study, performed in emergency ICU. Written informed consent was obtained from all enrolled patients or their next kin. All the procedures were done by anesthesiologists or pulmonologist with adequate training in bronchoscopy. The inclusion criteria included patients aged 18 to 75 years, requiring intubation or reintubation and mechanical ventilation in the ICU with an expected ventilation duration of more than 48 hours, and nonpregnant patients were eligible. Patients who had a previous tracheal injury, expected ventilation duration of less than 48 hour, pregnant patient, and patient admitted after cardiac arrest were excluded from the study.

Study group were divided into two groups. Group 1 was the CSSS group and group 2 was the ISSS group. Eligible patients were orally intubated with a special ET tube of $7.5 \mathrm{~mm}$ internal diameter called SACETT. The main objective of this study was to compare the effect of CSSS and ISSS on tracheal mucosa damage observed by fiberoptic bronchoscopy and on incidence of VAP.

The potential lesions on tracheal mucosa secondary to the cuff of the ET tube were not evaluated in this study. Only the front of the tracheal zone of the subglottic suctioning port was explored. The additional endpoints included the mean daily volume of suctioned secretions, the mean daily number of difficulties, the incidence of VAP, and the occurrence of postextubation laryngeal edema.

Group 1 patients received CSSS at $-20 \mathrm{~mm} \mathrm{Hg}$ and group 2 patients received ISSS at $-100 \mathrm{~mm} \mathrm{Hg}$ (during $15 \pm 3$ seconds and no suction during $8 \pm 3$ seconds) with suctioning unit. Computer-generated randomization was done.

\section{Evaluation of Tracheal Injury and Management of the Endotracheal Tube}

The degree of tracheal mucosa damage was displayed by using fiberoptic bronchoscopy after intubation (T0) and before extubation (T1). Hypothetically no injury observed at T0 but present at $\mathrm{T} 1$ or the worsening injury observed at $\mathrm{T} 0$ and exacerbating at T1 was studied. Bronchoscopy through the SACETT allowed a visualization of the tracheal mucosa in front of the suctioning port. The severity was graded into five categories (no injury, erythema, edema, ulceration, or necrosis). Fiberoptic bronchoscopy was performed by pulmonologists blinded for the study, with suctioning being stopped at the moment of the bronchoscopy. The exact position of the SACETT and the pressure of the cuff were checked daily and maintained between 22 and $28 \mathrm{~cm} \mathrm{H}_{2} \mathrm{O}$ to avoid inadvertent mobilization and under- or overinflation of cuff, respectively. If a tracheostomy was required, the bronchoscopy was performed before its realization.

\section{Diagnosis of Ventilator-Associated Pneumonia}

All randomized patients will be screened daily for the occurrence of clinical suspicion of VAP. Screening for VAP were maintained until the first episode of microbiologically confirmed VAP, or 48 hours after weaning from the ventilator or death, whichever occurred first. Patients requiring invasive mechanical ventilation within 48 hours of an attempt at extubation will be retained in the study.

Clinical suspicion of VAP will be based on the occurrence of pulmonary infection at least 48 hours after intubation and evidenced by the presence of a recent and persistent infiltrate on chest X-ray and at least two of the following criteria: fever $\left(>38.3^{\circ} \mathrm{C}\right)$ or hypothermia $\left(<36^{\circ} \mathrm{C}\right)$, leukocytosis $\left(>10.10^{9} / \mathrm{L}\right)$ or leukopenia $\left(<4.10^{9} / \mathrm{L}\right)$, and purulent tracheal secretions. Confirmation of pneumonia required a positive quantitative culture of either a protected telescoping catheter sample or bronchoalveolar lavage (BAL) fluid. Samples were also obtained before any change or initiation of antibiotic therapy. The empirical antibiotic therapy was guided by our existing hospital policy. VAP was confirmed if quantitative culture of the protected telescoping catheter sample or 
BAL fluid grew at least $10^{3}$ or $10^{4}$ colony-forming units $/ \mathrm{mL}$, respectively, of at least one microorganism. Microbiologist was blinded to the study groups.

Episodes of pneumonia diagnosed within 48 hours of ventilation onset were not considered ventilator-associated. A threshold of 5 days after initiation of mechanical ventilation will be used to distinguish early-onset ( $<5$ days) VAP and late-onset ( $\geq 5$ days) VAP. The rate of early-onset VAP was assessed in all randomized patients, whereas the rate of late-onset VAP was assessed in patients with at least 5 days of mechanical ventilation without early-onset VAP.

\section{Data Collection}

The following parameters were collected: age, sex, severity assessed according to the Simplified Acute Physiology Score II and the Sequential Organ Failure Assessment score during the 24 hours following intubation, reasons for ICU hospitalization, and intubation. Moreover, the number of intubations before randomization during the current hospitalization and the duration of intubation between $\mathrm{T} 0$ and $\mathrm{T} 1$ were recorded.

\section{Sample Size and Statistical Analysis}

Sample size of 140 patients (70 patients per group) was required for the study to have $90 \%$ power to show a $50 \%$ relative reduction of mucosal damage with ISSS at a one-sided $\alpha$ level of 0.05 . Statistical analysis was performed using SAS statistical software version 9.4 (SAS Institute, Cary, North Carolina, United States). Qualitative variables were compared between the two groups with the chi-square of the Fisher's exact test when needed, and continuous variables were compared using the Student's $t$-test or the Wilcoxon rank-sum test as appropriate. All $p$-values will be one-sided, and a $p$-value of $<0.05$ will be considered statistically significant.

\section{Results}

Total 140 participants were randomized between March 2021 and September 2021; 13 of them withdrew informed consent and thus 127 were included (CSSS, $n=64$ and ISSS, $n=63$ ). Baseline characteristics are provided in -Table 1. Of these, 38 participants had no fiberoptic bronchoscopy at $\mathrm{T} 1$, before extubating (20 and 18 in the CSSS and ISSS groups, respectively), as a result of unavailability of pulmonologist ( $n=10)$, self-extubating $(n=4)$, and death of the patient $(n=24)$. Consequently, 89 participants were analyzed on the primary endpoint (CSSS, $n=44$ and ISSS, $n=45$ ) (-Fig. 1). The occurrence or worsening of mucosal damage was observed in 20 (22.5\%) participants and did not differ between the two groups (CSSS, $n=12$ [27.3\%] vs. ISSS, $n=8$ [17.7\%], $p=0.365$ ). Individual data are presented in - Table 2 . No injury was seen at T0 in any group. Three participants developed local tracheal mucosa ulcerations in the CSSS group at T1.

The mean \pm standard deviation daily volume of suctioned secretion was significantly higher with ISSS as compared with CSSS (72 \pm 100 [interquartile range: 24-76] $\mathrm{mL}$ vs.
Table 1 Baseline characteristics

\begin{tabular}{|c|c|c|c|}
\hline & $\begin{array}{l}\text { Continuous } \\
\text { suctioning } \\
(n=64), n(\%)\end{array}$ & $\begin{array}{l}\text { Intermittent } \\
\text { suctioning } \\
(n=63), n(\%)\end{array}$ & $p$-Value \\
\hline Age $(y)$ & $60 \pm 15$ & $61 \pm 14$ & 0.711 \\
\hline Sex (male) & $36(56)$ & $42(65)$ & 0.556 \\
\hline \multicolumn{4}{|c|}{ Type of admission } \\
\hline Medical & $26(40.6)$ & $30(47.6)$ & \multirow[t]{4}{*}{0.182} \\
\hline $\begin{array}{l}\text { Unscheduled } \\
\text { surgery }\end{array}$ & $19(29.6)$ & $19(30.1)$ & \\
\hline $\begin{array}{l}\text { Scheduled } \\
\text { surgery }\end{array}$ & $8(12.5)$ & $6(9.5)$ & \\
\hline Trauma & $11(17.1)$ & $8(12.6)$ & \\
\hline SAPS II & $43 \pm 13$ & $46 \pm 14$ & 0.223 \\
\hline SOFA score & $7 \pm 5$ & $7 \pm 4$ & 0.894 \\
\hline \multicolumn{4}{|c|}{ Reason for intubation } \\
\hline $\begin{array}{l}\text { Respiratory } \\
\text { failure }\end{array}$ & $43(67)$ & $40(63.5)$ & \multirow[t]{4}{*}{0.734} \\
\hline Coma & $17(26)$ & $15(23.8)$ & \\
\hline Shock & $2(3)$ & $2(3.1)$ & \\
\hline Others & $2(3)$ & $6(9.5)$ & \\
\hline \multicolumn{4}{|c|}{ Number of intubation prior randomization } \\
\hline 0 & $18(28.1)$ & $23(36.5)$ & \multirow[t]{4}{*}{0.416} \\
\hline 1 & $35(54.6)$ & $31(49.2)$ & \\
\hline 2 & $10(15.6)$ & $8(12.7)$ & \\
\hline 3 & $1(1)$ & $3(4.7)$ & \\
\hline
\end{tabular}

Abbreviations: SAPS II, Simplified Acute Physiologic Score II; SOFA, Sequential Organ Failure Assessment.

$18 \pm 25$ [interquartile range: 7-31] $\mathrm{mL}$, respectively, $p<0.001$ ). Mean daily difficulties to aspirate through the suction lumen of the SACETT did not differ between the two groups (CSSS group, $0.38 \pm 0.52$ per day and ISSS group, $0.18 \pm 0.28$ per day, $p=0.102$ ), whereas mean daily negligible chance to aspirate was significantly higher with CSSS as compared with ISSS $(0.13 \pm 0.15$ per day vs. $0.03 \pm 0.06$ per day, respectively, $p<0.001)$. The duration of intubation between T0 and T1 did not differ between the CSSS and ISSS groups ( $12 \pm 7$ vs. $11 \pm 6$ days, $p=0.386$ ). Postextubation laryngeal edema was not observed in the two groups

The incidence of VAP did not differ between the CSSS and ISSS groups (5/64 [8\%] vs. 7/63 [11\%], $p=0.397)$.

\section{Discussion}

In our study, the total incidence of mucosa tracheal damage when subglottic secretion drainage was applied with the ET tube (SACETT) was $22.5 \%$, but the modality of suction (continuous suctioning vs. intermittent suctioning) did not alter the appearance of tracheal mucosa injuries. Seguin et al also observed 23\% mucosa tracheal damage in his study which was similar to our findings. ${ }^{1}$ Continuous suctioning was associated with a more frequent negligible chance to aspirate and lower volume of suctioned secretions. Postextubation laryngeal edema was not observed. This result has been 


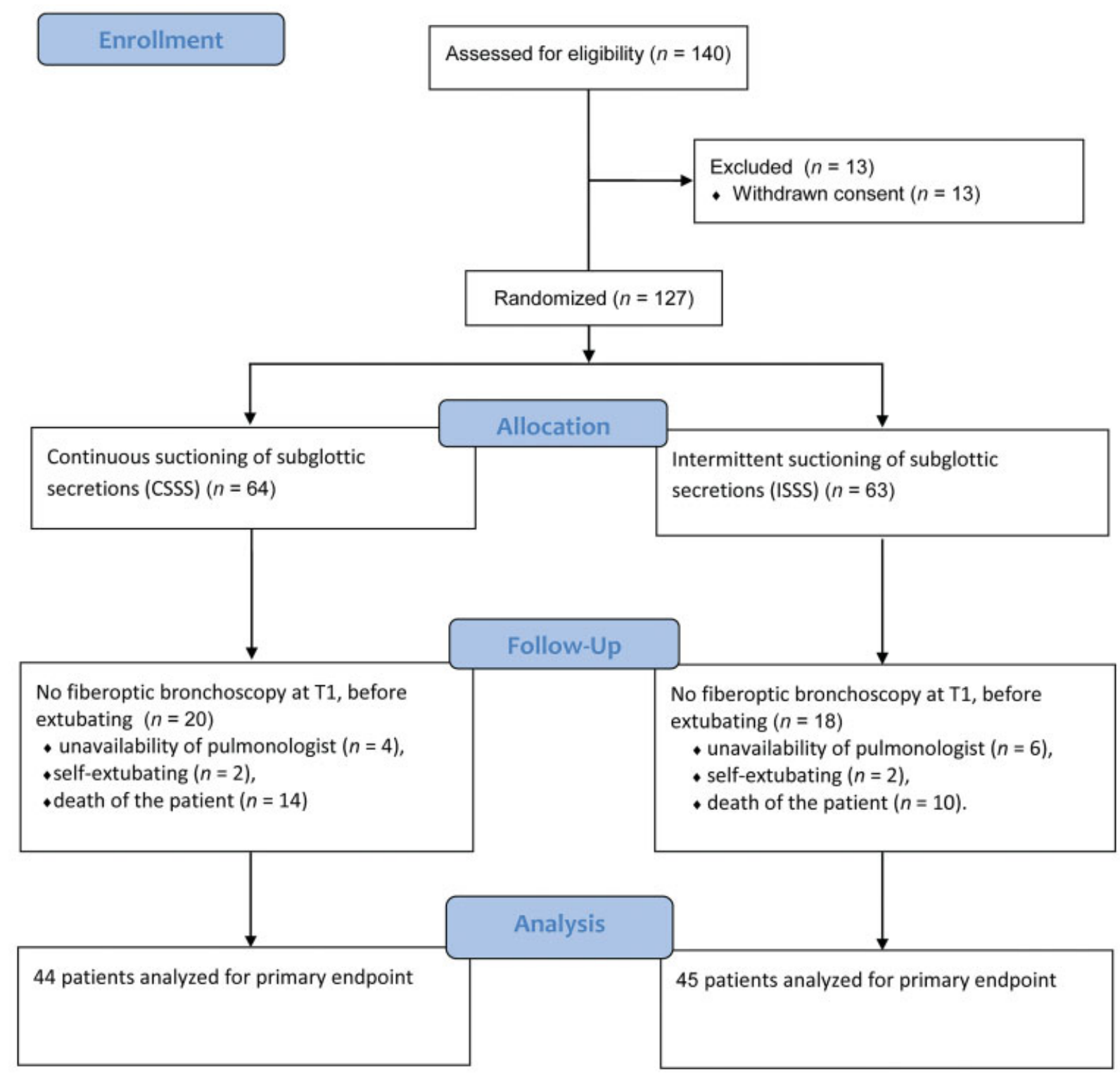

Fig. 1 Patients analyzed for the primary endpoint.

Table 2 Primary endpoint analysis of tracheal mucosa damage at T1

\begin{tabular}{|l|l|l|l|}
\hline $\begin{array}{l}\text { Type of } \\
\text { Injury }\end{array}$ & $\begin{array}{l}\text { Group 1 } \\
\text { (CSSS) } \\
\boldsymbol{n}=\mathbf{4 4}\end{array}$ & $\begin{array}{l}\text { Group 2 } \\
\text { (ISSS) } \\
\boldsymbol{n}=\mathbf{4 5}\end{array}$ & -Value \\
\hline No injury & $32(72.7)$ & $37(82.2)$ & 0.673 \\
\hline Erythema & $5(11.4)$ & $5(11.1)$ & 0.891 \\
\hline Edema & $4(9.1)$ & $2(4.4)$ & 0.573 \\
\hline Ulceration & $3(6.8)$ & $1(2.2)$ & 0.493 \\
\hline Necrosis & 0 & 0 & \\
\hline $\begin{array}{l}\text { Overall } \\
\text { injury }\end{array}$ & $12(27.3)$ & $8(17.7)$ & 0.365 \\
\hline
\end{tabular}

Abbreviations: CSSS, continuous suctioning of subglottic secretions; ISSS, intermittent suctioning of subglottic secretions.

Note: T1: bronchoscopy before extubation. analyzed in detail in the meta-analysis, but the absence of postextubation laryngeal edema does not mean that tracheal injuries induced by suctioning are not present. ${ }^{1-3}$

In a prospective randomized study performed in animals (sheep), Berra et al showed that CSSS at $\leq 20 \mathrm{~mm} \mathrm{Hg}$ with an 8-mm Hi-Lo Evac Mallinckrodt ET tube induced macroscopic tracheal injuries in all animals, especially necrosis and/or hemorrhage in the part of the subglottic port of the ET tube. ${ }^{5}$ However, best to mimic human tracheal orientation, it was not differentiated that the tracheal anatomy of sheep favored a tight contact between the subglottic port and tracheal mucosa, and our observations do not support this findings in humans.

In ICU patients, in older studies, it has been found that the incidence of $40 \%$ of postextubation laryngeal edema with continuous suctioning, was seen in only two of the five patients who were extubated. This was not significant. ${ }^{5}$ Before this publication, postextubation edema associated 
with continuous subglottic suctioning has been reported to be from 2.9 to $10.1 \%$ in larger populations but did not differ from control groups without suctioning. ${ }^{1,3}$ In our study, we do not find any postextubation laryngeal edema in any group. With regard to the high frequency of suction dysfunction, fiberoptic bronchoscope examination revealed that, in $85 \%$ of cases, dysfunction was attributed to an obstruction of the subglottic port related to suctioned mucosa raising concerns about the safety of the method. ${ }^{6}$ Two observations of tracheoesophageal fistula in severe trauma patients intubated with a Mallinckrodt Hi-Lo Evac and suspected to be in relation to the site of subglottic suctioning have been reported. ${ }^{7}$ In the same way, a recent report performed in six patients intubated with an 8-mm Mallinckrodt Hi-Lo Evac ET tube in which an intermittent suctioning at $-125 \mathrm{~mm} \mathrm{Hg}$ during 15 seconds was applied, showed on computed tomography scan an entrapment of the tracheal mucosa into the subglottic port of the tube. ${ }^{8,9}$ In our study, intermittent suctioning provided a significant threefold increase in volume of suctioned secretions, and this observation may be related to a lower rate of obstruction of the subglottic port. Although this point cannot be proved because it has not been specifically explored, this hypothesis is likely and reinforced by the higher rate of negligible chance to aspirate in the continuous suctioning group. This finding may be interesting in clinical practice because the more secretions are removed, the less will pass the ET cuff.

The expected tracheal mucosa damage was high but we observed that it was low as this can be explained by the utilization of the latest generation of ET tube SACETT used in our study in comparison to older studies. Evidently, in the latest generation, the subglottic port was closer to the cuff and the suction lumen larger limiting tracheal mucosa injury and obstruction, respectively. At the end, the $-100 \mathrm{~mm} \mathrm{Hg}$ applied in the intermittent group may appear very high, but the manufacturer recommended this level of aspiration. At least two studies have used such aspiration level. ${ }^{10-12}$

The incidence of VAP is lower in the CSSS group than the ICSS group ( $8 \%$ vs. $11 \%)$, and was not statistically significant in the current study. SACETT specialized tube is designed with a port above the cuff for aspirating the oropharyngeal secretions collected above the cuff, thereby preventing potential peritubular leakage, and reduces the incidence of VAP. To the best of our knowledge, this is the first study that has evaluated continuous suctioning versus intermittent subglottic suctioning for incidence of VAP. Jena et al compared suction above cuff and standard ET tubes in neurological patients for the incidence of VAP and found lower incidence with SACETT. However, the difference in incidence was not significant. ${ }^{13}$

\section{Conclusion}

On the basis of our result, the ISSS did not reduce mucosal damage as compared with CSSS. The aspirated volume was higher with ISSS. The incidence of VAP was lower with CSSS.
Source(s) of Support

None.

Presentation at a Meeting

No.

Organization

Place

Date

Conflict of Interest (If present, give more details)

None declared.

\section{References}

1 Seguin P, Le Pabic E, Tiercin M, et al. Effect of continuous versus intermittent subglottic suctioning on tracheal mucosa by the Mallinkrodt taper guard evac oral tracheal tube in intensive care unit ventilated patients. Indian J Crit Care Med 2018;22 (01):1-4

2 Damas P, Frippiat F, Ancion A, et al. Prevention of ventilatorassociated pneumonia and ventilator-associated conditions: a randomized controlled trial with subglottic secretion suctioning. Crit Care Med 2015;43(01):22-30

3 Muscedere J, Rewa O, McKechnie K, et al. Subglottic secretion drainage for the prevention of ventilator-associated pneumonia: a systematic review and meta-analysis. Crit Care Med $\cdots ; 20(39)$ : 198-591

4 Lacherade JC, De Jonghe B, Guezennec P, et al. Intermittent subglottic secretion drainage and ventilator-associated pneumonia: a multicenter trial. Am J Respir Crit Care Med 2010;182(07): 910-917

5 Berra L, De Marchi L, Panigada M, Yu ZX, Baccarelli A, Kolobow T. Evaluation of continuous aspiration of subglottic secretion in an in vivo study. Crit Care Med 2004;32(10):2071-2078

6 Girou E, Buu-Hoi A, Stephan F, et al. Airway colonisation in longterm mechanically ventilated patients. Effect of semi-recumbent position and continuous subglottic suctioning. Intensive Care Med 2004;30(02):225-233

7 Dragoumanis CK, Vretzakis GI, Papaioannou VE, Didilis VN, Vogiatzaki TD, Pneumatikos IA. Investigating the failure to aspirate subglottic secretions with the Evac endotracheal tube. Anesth Analg 2007;105(04):1083-1085

8 Harvey RC, Miller P, Lee JA, Bowton DL, MacGregor DA. Potential mucosal injury related to continuous aspiration of subglottic secretion device. Anesthesiology 2007;107(04):666-669

9 Spapen H, Suys E, Nieboer K, Stiers W, De Regt J. Automated intermittent aspiration of subglottic secretions and tracheal mucosa damage. Minerva Anestesiol 2013;79(03):316-317

10 Rello J, Soñora R, Jubert P, Artigas A, Rué M, Vallés J. Pneumonia in intubated patients: role of respiratory airway care. Am J Respir Crit Care Med 1996;154(01):111-115

11 Smulders K, van der Hoeven H, Weers-Pothoff I, VandenbrouckeGrauls C. A randomized clinical trial of intermittent subglottic secretion drainage in patients receiving mechanical ventilation. Chest 2002;121(03):858-862

12 Bouza E, Pérez MJ, Muñoz P, Rincón C, Barrio JM, Hortal J. Continuous aspiration of subglottic secretions in the prevention of ventilator-associated pneumonia in the postoperative period of major heart surgery. Chest 2008;134(05):938-946

13 Jena S, Kamath S, Masapu D, et al. Comparison of suction above cuff and standard endotracheal tubes in neurological patients for the incidence of ventilator-associated pneumonia and in-hospital outcome: a randomized controlled pilot study. Indian J Crit Care Med 2016;20(05):261-266 